\title{
Levels of serum biomarkers from a two- year multicentre trial are associated with treatment response on knee osteoarthritis cartilage loss as assessed by magnetic resonance imaging: an exploratory study
}

\author{
Johanne Martel-Pelletier', Jean-Pierre Raynauld ${ }^{1}$, François Mineau' ${ }^{1}$, François Abram², Patrice Paiement ${ }^{3}$,
} Philippe Delorme ${ }^{3}$ and Jean-Pierre Pelletier ${ }^{1 *}$

\begin{abstract}
Background: There is an obvious need to identify biomarkers that could predict patient response to an osteoarthritis $(\mathrm{OA})$ treatment. This post hoc study explored in a 2-year randomized controlled trial in patients with knee OA, the likelihood of some serum biomarkers to be associated with a better response to chondroitin sulfate in reducing cartilage volume loss.

Methods: Eight biomarkers were studied: hyaluronic acid (HA), C reactive protein (CRP), adipsin, leptin, Nterminal propeptide of collagen Ila (PIIANP), C-terminal crosslinked telopeptide of type I collagen (CTX-1), matrix metalloproteinase-1 (MMP-1), and MMP-3. Patients were treated with chondroitin sulfate (1200 mg/day; $n=57)$ or celecoxib (200 mg/day; $n=62$ ). Serum biomarkers were measured at baseline. The cartilage volume at baseline and its loss at 2 years were assessed by quantitative magnetic resonance imaging (MRI). Statistical analysis included analysis of covariance.
\end{abstract}

Results: As data from the original MOSAIC trial showed no differences in cartilage volume and loss in the lateral compartment of the knee joint between the two treatment groups in any comparison, only the medial compartment and its subregions were studied. Stratification according to the median biomarker levels was used to discriminate treatment effect. In patients with levels of biomarkers of inflammation (HA, leptin and adipsin) lower than the median, those treated with chondroitin sulfate demonstrated less cartilage volume loss in the medial compartment, condyle, and plateau $(p \leq 0.047)$. In contrast, patients treated with chondroitin sulfate with higher levels of MMP-1 and MMP-3, biomarkers of cartilage catabolism, had less cartilage volume loss in the medial compartment, condyle, and plateau $(p \leq 0.050)$. Patients with higher levels of PIIANP and CTX-1, biomarkers related to collagen anabolism and bone catabolism, respectively, had reduced cartilage volume loss in the medial condyle $(p \leq 0.026)$ in the chondroitin sulfate group.

Conclusion: This study is suggestive of a potentially greater response to chondroitin sulfate treatment on cartilage volume loss in patients with knee OA with low level of inflammation and/or greater level of cartilage catabolism.

(Continued on next page)

\footnotetext{
* Correspondence: dr@jppelletier.ca

'Osteoarthritis Research Unit, University of Montreal Hospital Research Centre

(CRCHUM), 900 Saint-Denis, Suite R11.412, Montreal, Quebec H2X 0A9,

Canada

Full list of author information is available at the end of the article
} 
(Continued from previous page)

Trial registration: This is a post hoc study. Original trial registration: ClinicalTrials.gov, NCT01354145. Registered on 13 May 2011.

Keywords: Knee, Osteoarthritis, Biomarkers, Clinical trial

\section{Background}

Osteoarthritis (OA) is one of the most highly prevalent joint diseases characterized by progressive articular structural changes and cartilage degradation. This disease represents an increasing burden from a medical, social and economic point of view, as it affects the majority of people in the second half of their lifespan, having a significant negative impact on quality of life. The primary etiological factors may be numerous; hence, in the majority of patients the disease is considered to be idiopathic. At present, pharmacologic therapies only reduce symptoms, and they have adverse events. For this reason, attention has been focused on the investigation and development of new types of drugs that in addition to improving the clinical symptoms of OA and having better safety profiles can also decrease cartilage volume loss.

One such product is chondroitin sulfate, which is a sulfated glycosaminoglycan that has been shown to play a role not only in the metabolism of the proteoglycans (a major macromolecule in the cartilage), but also in OA joint tissues and cells, to improve the anabolic/catabolic balance of the extracellular cartilage matrix, and to reduce some pro-inflammatory and catabolic factors and the resorptive properties of subchondral bone osteoblasts [1-5]. In addition to its ability to reduce OA symptoms, similar to that of non-steroidal anti-inflammatory drugs (NSAIDs) [6, 7], in clinical trials and studies $[6,8,9]$ it has also been shown to decrease cartilage volume loss as assessed by quantitative magnetic resonance imaging (qMRI). However, as mentioned in the original trial publication [6], although it has been recommended in some guidelines $[10,11]$, a general consensus has not been reached on the use of chondroitin sulfate [12, 13], due in part to the unavailability of prescription-quality products, indicated for use in OA, which have been evaluated by the US Food and Drug Administration (FDA) [12]. Over the years, several studies have looked into identifying biomarkers with predictive value to assess the risk of OA progression, particularly in patients with knee OA (see reviews [14-18]). A number of such biomarkers have been identified, predominantly in observational patient cohort studies, yet none has qualified for use in clinical practice for the follow up of patients with knee OA $[14,19]$. There is an obvious need to identify prognostic biomarkers, but an even greater need to identify biomarkers that could predict patient response to potential disease-modifying OA drug treatments $[14,16,20,21]$.
Recently, the Osteoarthritis Research Society InternationalFDA (OARSI-FDA) Biomarkers Group identified twelve distinct biochemical biomarkers of bone and cartilage turnover that could be of interest and included in the BIPEDS classification system [17]. This group further looked at the predictive validity of eighteen biomarkers as OA-related predictors of radiographic and persistent pain progression [18]. They identified nine such biomarkers that were individually able to predict clinically relevant progression over 48 months; eight catabolic and one anabolic biomarker.

Most studies assessing biomarkers in relation to articular changes have used radiographic parameters, a technology that is not sensitive for the validation of biomarkers $[14,19,20]$. Converging data argue for the use of qMRI, another imaging technology that may assist in delineating the predictive value of biomarkers with regard to OA joint structural changes [14, 16, 22]. A few trials have used this technology to identify biomarkers that would be of value in predicting drug efficacy in cartilage volume loss and clinical outcomes such as total knee replacement $[23,24]$.

The present study aimed to explore whether biomarkers could identify patients with knee OA most likely to benefit from a chondroitin sulfate treatment, a commonly used treatment for knee OA. The information gathered from the present study could be very useful in the context of personalized medicine, as targeting responders would reduce the cost of treatment for OA patients and, importantly, improve patient benefits.

Using the knee OA patient cohort from a phase III clinical trial [6], eight serum biomarkers ( $C$ reactive protein (CRP), hyaluronic acid (HA), leptin, adipsin, matrix metalloproteinase-1 (MMP-1), MMP-3, Nterminal propeptide of collagen II $\alpha$ (PIIANP) and Cterminal crosslinked telopeptide of type I collagen $(\mathrm{CTX}-1)$ ) were assessed for their predictive value in determining the evolution of changes in cartilage in OA over a 2 -year period by qMRI, and in relation to the effect of chondroitin sulfate treatment on cartilage volume loss. The rationale behind the selection of biomarkers was to use those that are representative of the most important pathways related to OA progression: inflammation (CRP, HA, leptin, and adipsin), cartilage catabolism (MMP-1, MMP-3) and anabolism (PIIANP), and bone remodeling (CTX-1), which have been found promising $[16-18,23,24]$. We reasoned that the biomarkers 
chosen for the study covered a broad range of pathways including the major pathological pathways of $\mathrm{OA}$ and were therefore likely to provide new information about biomarkers that could be associated with a treatment effect on cartilage volume loss.

\section{Methods}

\section{Patients and treatments}

This post hoc study used the patient cohort from a 2year phase-III comparative, double-blind clinical trial (ClinicalTrials.gov Identifier NCT01354145) [6]. In brief, patients with symptomatic knee OA, diagnosed according to the clinical and radiological criteria of the American College of Rheumatology [25], and clinical synovitis, were randomized to explore the effects of chondroitin sulfate (Condrosan'; Bioibérica S.A.U., Barcelona, Spain) (1200 mg daily; $n=97)$ compared to celecoxib (200 mg daily; $n=97$ ) on cartilage volume loss in knee OA. Celecoxib was chosen as the comparator since, among many other reasons, it was shown to have no effect on the progression of cartilage volume loss in patients with knee OA [26].

The original study protocol was approved by a central review board (Institutional Review Board (IRB) Services, Toronto, ON, Canada) and the IRB of the Centre hospitalier de l'Université de Sherbrooke (CHUS), Sherbrooke, QC, Canada. The original trial [6] was conducted in compliance with the ethical principles that have their origin in the Declaration of Helsinki (2000) and are consistent with "Good Clinical Practice" International Conference of Harmonization (ICH) Tripartite Guideline (January 1997) and the applicable laws and regulations of Canada, whichever afforded the greater protection to the individual. Ethical approval for this post hoc study was obtained with the original study, thus further approval was not required. All patients provided informed consent.

The according-to-protocol (ATP) population (chondroitin sulfate $n=57$; celecoxib $n=62$ ) was chosen as it included patients who fully complied with the study's 2-year protocol and for whom serum and MRI at baseline and at 2 years were available. Of note, compared to the original ATP population [6], one serum sample was missing in the celecoxib group. These patients had taken the study medication throughout the entire study period (compliance $\geq 75 \%$ ) and had no major protocol violations.

\section{Outcome}

The primary outcome of the study was the usefulness of some serum biomarkers to indicate patients who are most likely to have reduction in cartilage volume loss in response to chondroitin sulfate. Of note, pain was not considered as an outcome because in the original MOSAIC trial [6] both therapeutic groups experienced a comparable reduction in disease-associated pain at 2 years. Moreover, data from the original trial [6] showed no difference between the two treatment groups in cartilage volume in the lateral compartment of the knee at baseline or loss of cartilage volume in any comparison; therefore, only the medial compartment of the knee (condyle plus plateau) and its sub-regions (condyle and plateau) were studied.

\section{Clinical, structural and biomarker assessments Clinical evaluation at baseline}

Clinical evaluation at baseline is shown in Table 1.

Clinical evaluation at baseline included assessment of knee pain on a visual analog scale (VAS), the Western Ontario and McMaster Universities Osteoarthritis Index (WOMAC) scores, the quality of life Short Form 36 General Health (SF-36), and clinical examination (swelling, visual examination; effusion, bulge sign).

\section{MRI evaluation at baseline}

Assessment using MR images included, in addition to cartilage volume [27, 28] (see subsequent description), the extent of synovitis assessed by measuring synovial thickness $(\mathrm{mm})$ in four regions of interest (ROIs): the medial and lateral articular recess and the medial and lateral outer wall of the suprapatellar bursa [29]. The synovial fluid was determined using a fully automated system as previously described [30]. Bone marrow lesions were assessed in the same MRI sequences used for the cartilage assessment [31], and their extent was evaluated using the following scale: 0 , absence; $1,<25 \% ; 2$, $25-50 \% ; 3,>50 \%$ of the surface. The readers were blinded to treatment and to MRI examination time point except for baseline [6].

\section{Cartilage volume assessment}

Cartilage volume was assessed by MRI, which was performed on $1.5 \mathrm{~T}$ scanners (Siemens, Erlangen, Germany; General Electric, Milwaukee, WI, USA) using a standard knee coil, and the sequence acquisitions were as previously described [32]. The cartilage volume was measured by two experienced readers trained by musculoskeletal radiologists using the computer program Cartiscope ${ }^{\text {th }}$ (ArthroLab, Montreal, QC, Canada) as previously described $[27,28]$. The change (percentage) in knee cartilage volume was obtained by subtracting the follow-up volume from the initial (baseline) volume divided by the initial (baseline) volume multiplied by 100. The percent coefficient of variation $(\mathrm{CV} \%)$ is excellent as described [27].

\section{Biomarker assessment}

Blood samples were obtained after subjects fasted overnight. The samples were first allowed to coagulate and 
Table 1 Patient characteristics

\begin{tabular}{|c|c|c|c|}
\hline & Chondroitin sulfate $(n=57)$ & Celecoxib $(n=62)$ & $P$ value \\
\hline Male, $n(\%)$ & $26(46)$ & $29(47)$ & $0.899^{*}$ \\
\hline Age, years & $61 \pm 8$ & $61 \pm 8$ & 0.909 \\
\hline Body mass index, $\mathrm{kg} / \mathrm{m}^{2}$ & $30.3 \pm 6.42$ & $32.5 \pm 5.9$ & 0.020 \\
\hline Synovial membrane thickness, $\mathrm{mm}$ & $1.04 \pm 0.23$ & $1.01 \pm 0.22$ & 0.528 \\
\hline Bone marrow lesion, score & $2.49 \pm 3.20$ & $2.63 \pm 2.33$ & 0.291 \\
\hline Synovial fluid volume, $\mathrm{mL}$ & $14.8 \pm 15.9$ & $11.2 \pm 12.3$ & 0.210 \\
\hline Joint swelling and effusion, $n(\%)$ & $37(65)$ & $32(52)$ & $0.142^{*}$ \\
\hline Pain VAS, mm & $60.6 \pm 15.9$ & $57.2 \pm 18.6$ & 0.252 \\
\hline \multicolumn{4}{|l|}{ WOMAC } \\
\hline Total score $(0-240)$ & $121 \pm 38$ & $120 \pm 46$ & 0.934 \\
\hline Pain score (0-50) & $24 \pm 8$ & $24 \pm 9$ & 0.909 \\
\hline Stiffness score (0-20) & $11 \pm 4$ & $11 \pm 4$ & 0.694 \\
\hline Physical function score (0-170) & $86 \pm 29$ & $85 \pm 34$ & 0.842 \\
\hline \multicolumn{4}{|l|}{ Quality of life (SF-36) } \\
\hline Physical component summary & $36.31 \pm 7.82$ & $36.33 \pm 8.13$ & 0.610 \\
\hline \multicolumn{4}{|l|}{ Cartilage volume $\left(\mathrm{mm}^{3}\right)$} \\
\hline \multicolumn{4}{|l|}{ Medial } \\
\hline Compartment & $4586 \pm 1517$ & $4324 \pm 1309$ & 0.445 \\
\hline Condyle & $2881 \pm 1020$ & $2682 \pm 833$ & 0.448 \\
\hline Plateau & $1705 \pm 545$ & $1642 \pm 532$ & 0.529 \\
\hline
\end{tabular}

were then centrifuged (2200 rpm/1000 g, 10 minutes). Samples were stored at $-80{ }^{\circ} \mathrm{C}$ until analyzed.

CTX-1 (Neo Scientific, Cambridge, MA, USA), HA (R\&D Systems, Minneapolis, MN, USA) and PIIANP (EMD Millipore Corporation, Billerica, MA, USA) were assessed using specific ELISAs. Each assay is highly sensitive (sensitivity for CTX-1, $0.1 \mathrm{ng} / \mathrm{mL}$; HA, $0.027 \mathrm{ng} / \mathrm{mL}$; and PIIANP, $30.0 \mathrm{ng} / \mathrm{mL}$ ) and designed to eliminate interference by other factors present in biological samples. Factors were read with a spectrophotometer (Multiskan Spectrum; Thermo Fisher Scientific, Mississauga, ON, Canada) and analyses performed with SkanIt sofware (Thermo). The CRP data (absolute values) available from the clinical trial report were used. The levels of the adipokines leptin and adipsin, and of MMP-1 and MMP-3 were assessed using the Human Magnetic Luminex Performance Assay kits (R\&D Systems) in accordance with the manufacturer's instructions with the minimum detectable dose (MDD) of $7.69 \mathrm{ng} / \mathrm{mL} ; 1.8 \mathrm{pg} / \mathrm{mL} ; 0.57 \mathrm{pg} / \mathrm{mL}$ and $1.8 \mathrm{pg} / \mathrm{mL}$, respectively. The assessment was performed using a multiplex immunoassay (Luminex Corporation, Austin, TX, USA) on a LiquiChip 200. Analyte-specific antibodies were read using a Luminex analyzer, which discriminates each different analyte, and quantitated using LiquidChip
Analyzer (QIAGEN, Toronto, ON, Canada). For each factor, an 8-point standard curve and appropriate controls were included, and samples were done in duplicate.

\section{Statistical analyses}

Descriptive variables at baseline were presented as number, percentage, or mean \pm standard deviation (SD) (Table 1). Differences between the two treatment groups were assessed using Student's $t$ test or the Mann-Whitney test for continuous variables with a non-normal distribution, and the chi-squared or Fisher's exact test for categorical variables (Tables 1 and 2). Correlation between all biomarker levels at baseline were carried out using Pearson's correlation analysis in all subjects (Table 3).

Linear regressions adjusting for age, gender and body mass index (BMI) were also carried out to assess associations between baseline serum levels of the biomarkers and cartilage volume at baseline, and volume loss over 2 years within the medial compartment, medial condyle and medial plateau in all subjects (Table 4).

Since serum biomarkers are relatively new to the OA field in the context of a clinical trial, the median baseline biomarker levels were used to discriminate for the overall cohort (Additional file 1: Table S1) and within 
Table 2 Biomarker levels at baseline

\begin{tabular}{llll}
\hline & Chondroitin sulfate $(n=57)$ & Celecoxib $(n=62)$ & $P$ value \\
\hline CRP, $\mu g / \mathrm{mL}^{+}$ & $4.02 \pm 5.41(n=48)$ & $5.56 \pm 5.54(n=46)$ & 0.042 \\
$\mathrm{HA}, \mathrm{ng} / \mathrm{mL}$ & $62.46 \pm 44.81$ & $72.46 \pm 60.45$ & 0.267 \\
Leptin, $\mathrm{ng} / \mathrm{mL}$ & $37.60 \pm 43.78$ & $44.23 \pm 38.53$ & 0.214 \\
Adipsin, $\mu \mathrm{g} / \mathrm{mL}$ & $4.05 \pm 1.07$ & $4.19 \pm 1.07$ & 0.638 \\
MMP-1, $\mathrm{ng} / \mathrm{mL}$ & $5.11 \pm 4.13$ & $4.69 \pm 3.15$ & 0.827 \\
MMP-3, ng/mL & $13.03 \pm 6.31$ & $13.85 \pm 11.04$ & 0.720 \\
PIIANP, $\mu \mathrm{g} / \mathrm{mL}$ & $4.10 \pm 1.92$ & $4.10 \pm 1.61$ & 0.468 \\
CTX-1, ng/mL & $0.59 \pm 0.18$ & $0.53 \pm 0.12$ & 0.035 \\
\hline
\end{tabular}

Data shown are mean \pm SD. CRP C reactive protein, HA hyaluronic acid, MMP matrix metalloproteinase, PIIANP N-terminal propeptide of collagen Ila, CTX-1 Cterminal crosslinked telopeptide of type I collagen. ${ }^{*}$ Mann-Whitney test; $p$ values in italics are statistically significant. ${ }^{\dagger}$ Data missing for 25 patients at baseline

the treatment groups (chondroitin sulfate vs. celecoxib) (Table 5, Additional file 1: Table S2) to assess associations with baseline cartilage volume (medial compartment, medial condyle and medial plateau) and the predictive power of change in cartilage volume in the same regions at 2 years. The difference between mean cartilage volume within the three knee areas for lower vs. higher median biomarker levels was assessed by univariate analysis using Student's $t$ test or the MannWhitney test if the distribution was non-normal distribution (Additional file 1: Table S2).

Multivariate analyses (ANCOVA) were performed adjusting for the potential confounding factors at the onset of the study, including age, gender, BMI, and baseline cartilage volume for HA only (Table 5), as it is the only biomarker that showed, in patients with levels lower than the median, a statistically significant difference in the medial condyle between treatment groups (see Additional file 1: Table S2).

All tests were two-sided and a $p$ value $<0.05$ was considered statistically significant. Since this was an exploratory study, no statistical corrections were made for multiple comparisons. All statistical analyses were performed using SAS software, version 9.3 (SAS Institute, Cary, NC, USA).

\section{Results}

\section{Baseline demographic, clinical, and imaging data}

The two therapeutic groups were balanced with the exception of BMI, which was slightly higher in the

Table 3 Correlations between the biomarker levels at baseline in all subjects

\begin{tabular}{|c|c|c|c|c|c|c|c|c|c|}
\hline & & $\begin{array}{l}\text { CRP } \\
\left(n=94^{*}\right) \\
\end{array}$ & $\begin{array}{l}\text { HA } \\
(n=119)\end{array}$ & $\begin{array}{l}\text { Leptin } \\
(n=119)\end{array}$ & $\begin{array}{l}\text { Adipsin } \\
(n=119)\end{array}$ & $\begin{array}{l}\text { MMP-1 } \\
(n=119)\end{array}$ & $\begin{array}{l}\text { MMP-3 } \\
(n=119)\end{array}$ & $\begin{array}{l}\text { PIIANP } \\
(n=119)\end{array}$ & $\begin{array}{l}\text { CTX-1 } \\
\left(n=117^{* *}\right)\end{array}$ \\
\hline \multirow[t]{2}{*}{ CRP } & $r$ & - & - & - & - & - & - & - & - \\
\hline & $p$ value ${ }^{\dagger}$ & - & - & - & - & - & - & - & - \\
\hline \multirow[t]{2}{*}{$\mathrm{HA}$} & $r$ & $<0.0001$ & - & - & - & - & - & - & - \\
\hline & $p$ value & $>0.999$ & - & - & - & - & - & - & - \\
\hline \multirow[t]{2}{*}{ Leptin } & $r$ & 0.344 & -0.006 & - & - & - & - & - & - \\
\hline & $p$ value & 0.001 & 0.947 & - & - & - & - & - & - \\
\hline \multirow[t]{2}{*}{ Adipsin } & $r$ & -0.016 & 0.188 & 0.343 & - & - & - & - & - \\
\hline & $p$ value & 0.881 & 0.040 & 0.001 & - & - & - & - & - \\
\hline \multirow[t]{2}{*}{ MMP-1 } & $r$ & 0.045 & -0.009 & 0.036 & 0.234 & - & - & - & - \\
\hline & $p$ value & 0.664 & 0.922 & 0.702 & 0.010 & - & - & - & - \\
\hline \multirow[t]{2}{*}{ MMP-3 } & $r$ & 0.051 & 0.195 & -0.245 & 0.049 & -0.064 & - & - & - \\
\hline & $p$ value & 0.627 & 0.033 & 0.007 & 0.594 & 0.489 & - & - & - \\
\hline \multirow[t]{2}{*}{ PIIANP } & $r$ & 0.025 & -0.042 & 0.015 & -0.059 & -0.277 & -0.070 & - & - \\
\hline & $p$ value & 0.813 & 0.650 & 0.875 & 0.524 & 0.002 & 0.451 & - & - \\
\hline \multirow[t]{2}{*}{ CTX-1 } & $r$ & -0.093 & 0.063 & 0.016 & -0.105 & -0.049 & -0.099 & 0.075 & - \\
\hline & $p$ value & 0.380 & 0.497 & 0.862 & 0.262 & 0.601 & 0.287 & 0.422 & - \\
\hline
\end{tabular}

CRP C reactive protein, $H A$ hyaluronic acid, MMP matrix metalloproteinase, PIIANP N-terminal propeptide of collagen Ila, CTX-1 C-terminal crosslinked telopeptide of type I collagen. *Data missing for 25 patients at baseline; ${ }^{* *}$ data missing for 2 patients at baseline

${ }^{\dagger}$ Pearson's correlation; $p$ values in italics are statistically significant 
Table 4 Associations between cartilage volume and its loss and biomarker levels at baseline for all subjects

\begin{tabular}{|c|c|c|c|c|c|c|c|c|c|}
\hline & & $\begin{array}{l}\text { CRP } \\
\left(n=94^{*}\right)\end{array}$ & $\begin{array}{l}\mathrm{HA} \\
(n=119)\end{array}$ & $\begin{array}{l}\text { Leptin } \\
(n=119)\end{array}$ & $\begin{array}{l}\text { Adipsin } \\
(n=119)\end{array}$ & $\begin{array}{l}\text { MMP-1 } \\
(n=119) \\
\end{array}$ & $\begin{array}{l}\text { MMP-3 } \\
(n=119)\end{array}$ & $\begin{array}{l}\text { PIIANP } \\
(n=119)\end{array}$ & $\begin{array}{l}\text { CTX-1 } \\
\left(n=117^{* *}\right)\end{array}$ \\
\hline \multicolumn{10}{|c|}{ Cartilage volume at baseline } \\
\hline \multirow[t]{2}{*}{ Medial compartment } & $\beta$ & -49.24 & -1.66 & -4.26 & 101.26 & -2.49 & 9.35 & -40.65 & -773.37 \\
\hline & $p$ value $^{\dagger}$ & 0.045 & 0.409 & 0.385 & 0.315 & 0.931 & 0.454 & 0.489 & 0.256 \\
\hline \multirow[t]{2}{*}{ Medial condyle } & $\beta$ & -37.09 & -0.75 & -2.62 & 74.50 & -1.99 & 7.41 & -38.66 & -407.44 \\
\hline & $p$ value & 0.030 & 0.591 & 0.441 & 0.287 & 0.920 & 0.392 & 0.342 & 0.389 \\
\hline \multirow[t]{2}{*}{ Medial plateau } & $\beta$ & -12.15 & -0.91 & -1.63 & 26.77 & -0.50 & 1.94 & -1.99 & -365.94 \\
\hline & $p$ value & 0.170 & 0.215 & 0.361 & 0.468 & 0.962 & 0.671 & 0.926 & 0.141 \\
\hline \multicolumn{10}{|c|}{ Cartilage volume loss at 2 years } \\
\hline \multirow[t]{2}{*}{ Medial compartment } & $\beta$ & -0.179 & 0.001 & 0.009 & 0.334 & 0.049 & -0.022 & -0.546 & 3.418 \\
\hline & $p$ value & 0.029 & 0.905 & 0.590 & 0.342 & 0.622 & 0.615 & 0.007 & 0.150 \\
\hline \multirow[t]{2}{*}{ Medial condyle } & $\beta$ & -0.184 & 0.004 & -0.012 & 0.275 & 0.137 & -0.038 & -0.606 & 2.792 \\
\hline & $p$ value & 0.050 & 0.643 & 0.560 & 0.501 & 0.235 & 0.455 & 0.010 & 0.313 \\
\hline \multirow[t]{2}{*}{ Medial plateau } & $\beta$ & -0.164 & -0.005 & 0.049 & 0.415 & -0.100 & -0.001 & -0.420 & 4.532 \\
\hline & $p$ value & 0.102 & 0.512 & 0.016 & 0.321 & 0.402 & 0.989 & 0.083 & 0.106 \\
\hline
\end{tabular}

CRP C reactive protein, HA hyaluronic acid, MMP matrix metalloproteinase, PIIANP N-terminal propeptide of collagen Ila, CTX-1 C-terminal crosslinked telopeptide of type I collagen, $\beta$ regression parameter estimate of the biomarker. ${ }^{*}$ Data missing for 25 patients at baseline; ${ }^{* *}$ data missing for 2 patients at baseline. ${ }^{\dagger}$ Linear regression adjusted for age, gender and body mass index; $p$ values in italics are statistically significant

celecoxib group than in the chondroitin sulfate group (Table 1).

\section{Biomarkers \\ Baseline levels}

In the study population, with the exception of lower values of CRP and higher values of CTX-1 in the chondroitin sulfate group, there was no significant difference between the two groups (Table 2). The differences in the levels of CTX-1, although statistically significant, were very small and unlikely to be clinically relevant. Moreover, those differences found for CRP and CTX-1 no longer existed when the data were stratified by the median value (data not shown).

\section{Correlations between biomarker levels}

There was positive correlation between levels (Table 3) of CRP and leptin, HA and adipsin, HA and MMP-3, leptin and adipsin, and adipsin and MMP-1. There was negative correlation between MMP-1 and PIIANP, and between leptin and MMP-3.

\section{Associations between biomarkers and cartilage volume}

A statistically significant negative adjusted (age, gender, BMI) linear association was found between the level of CRP and cartilage volume in the medial compartment and condyle at baseline $(p=0.045$ and $p=0.030$, respectively) (Table 4). A statistically significant negative adjusted association was also found between the levels of CRP and PIIANP and cartilage volume loss: medial compartment $(p=0.029$ and $p=0.007$, respectively) and condyle ( $p=0.050$ and $p=0.010$, respectively). A statistically significant positive adjusted association was found between the level of leptin and cartilage volume loss in the plateau $(p=0.016)$.

\section{Biomarker and cartilage volume levels at baseline and treatment effects}

Since this work is among the first attempts to assess the impact of the studied biomarkers on knee OA structure as assessed by qMRI in relation to OA treatment, and no a priori cutoff values were available, we elected, as mentioned in "Statistical analysis", to stratify the biomarkers at baseline according to their median levels. First, there were no differences in the cartilage volume between the two stratifications for any of the biomarkers (Additional file 1: Table S1). There were also no significant differences between the two treatment groups for each biomarker with the exception of HA, which, at baseline levels lower than the median, showed a statistically significant increase $(p=0.030)$ in volume for chondroitin sulfate vs. celecoxib (Additional file 1: Table S2).

Of all of the biomarkers studied (Table 5), only CRP did not show any difference in the extent of cartilage volume loss between the treatment groups, in either the lower or higher median group. The levels of all the other biomarkers were able to discriminate the extent of cartilage degradation between the treatment groups.

With regard to the biomarkers of inflammation (Table 5), compared to celecoxib, patients with levels of HA lower than the median treated with chondroitin sulfate demonstrated less cartilage volume loss in the medial 
Table 5 Two-year medial region cartilage volume loss (\%) according to median value of baseline biomarker levels per treatment group

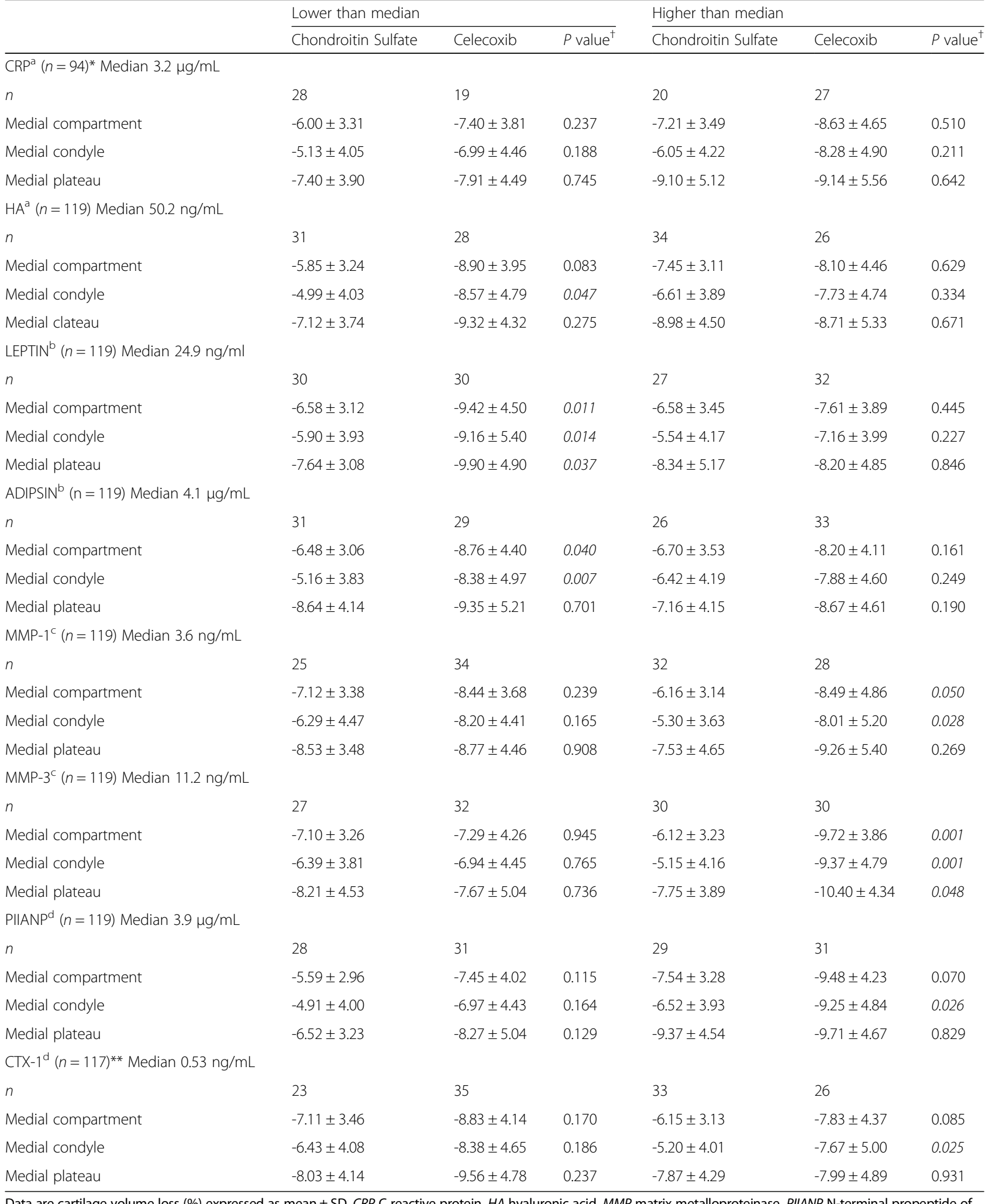

Data are cartilage volume loss (\%) expressed as mean \pm SD. CRP C reactive protein, HA hyaluronic acid, MMP matrix metalloproteinase, PIIANP N-terminal propeptide of collagen Ila, CTX-1 C-terminal crosslinked telopeptide of type I collagen. Biomarkers related to ainflammation; ${ }^{b}$ adipokines; ' ${ }^{C}$ matrix metalloproteinases; ${ }^{d}$ collagen metabolism. ${ }^{\dagger}$ Analysis of covariance adjusted for age, gender, body mass index and, for HA only, cartilage volume at baseline; $p$ values in italics are statistically significant. *Data missing for 25 patients at baseline; ${ }^{* *}$ data missing for 2 patients at baseline 
condyle $(p=0.047)$, with a numerical trend toward significance in the compartment $(p=0.083)$.

Similarly, the lower levels of the two studied adipokines (Table 5), factors involved in inflammation and in cartilage catabolism, showed differentiation between the two treatment groups, with patients treated with chondroitin sulfate having less cartilage volume loss than with celecoxib. This was found in all medial sub-regions for leptin $(p \leq 0.037)$, and in the compartment $(p=0.040)$ and condyle $(p=0.007)$ for adipsin.

In contrast, for MMP-1 and MMP-3 (Table 5), it was in patients with the higher levels that differences between the two treatment groups were found: for MMP-1, patients treated with chondroitin sulfate had less cartilage volume loss than with celecoxib in the medial compartment $(p=0.050)$ and condyle $(p=0.028)$, and for MMP-3 in the medial compartment $(p=0.001)$ and both the condyle $(p=0.001)$ and plateau $(p=0.048)$.

For PIIANP and CTX-1 (Table 5), which are biomarkers related to collagen anabolism and catabolism, respectively, patients with higher baseline levels that were treated with chondroitin sulfate had less cartilage volume loss in the medial condyle compared to the celecoxib group $(p \leq 0.026)$.

\section{Discussion}

Disease-modifying OA drug trials would benefit from the availability of biomarkers that could predict patients who are most likely to be responsive to a given treatment. The present study, although exploratory, provides important and novel information about the association of some serum biomarker levels with cartilage degradation, which could help to identify such patients. The original trial [6] demonstrated that chondroitin sulfate treatment in patients with knee OA had a significant beneficial effect on cartilage volume loss in the medial compartment (condyle). Here, we further showed that higher baseline values of the biomarkers PIIANP, CTX1, MMP-1, and MMP-3, and lower baseline values of HA, leptin and adipsin seem to be associated with response to chondroitin sulfate treatment in reducing cartilage loss in patients with knee OA.

Data first showed that the extent of systemic inflammation at baseline, assessed by the levels of CRP and HA, positively correlated with the levels of cartilage catabolic factors such as leptin, adipsin, and MMP-3. Moreover, the level of leptin, an adipokine associated with inflammation, was found to positively correlate with the level of another adipokine, adipsin, which was also shown to be associated with cartilage degradation [24]. Interestingly, the inverse correlation between the levels of PIIANP and MMP-1 could likely reflect increased turnover and loss of type II collagen in cartilage, as PIIANP is associated with collagen formation/repair and MMP-1 with this macromolecule's degradation. From these data, it can be hypothesized that joint inflammation often seen in knee OA and reflected by an increased level of CRP or HA, may induce an increase in the level of leptin and, secondarily, catabolic factors such as adipsin and MMPs [33], leading to cartilage degradation and loss, the latter reflected by negative correlation with PIIANP levels. Such a hypothesis is supported by the findings of this study, which show a negative association between the level of CRP at baseline and the cartilage volume at baseline, and cartilage loss at 2 years. This is well in line with a recent review on several related studies [34]. Our findings also concur with previous reports in which the level of CRP is correlated with disease progression in patients with knee OA assessed by X-rays and MRI, and an elevated level is associated with poorer disease outcome [23, 35-38]. However, these findings have not yet attained unanimity [39].

This study also showed that lower baseline values of leptin, adipsin and $\mathrm{HA}$, and higher values of MMP-3 and MMP-1, were associated with greater reduction in cartilage volume loss with chondroitin sulfate than celecoxib. This suggests that patients with a milder level of inflammation, yet sufficient to induce the synthesis of cartilage catabolic factors (MMPs), and greater cartilage catabolism and disease progression [23], are more likely to be responsive to treatment with chondroitin sulfate. These findings are in line with those of previous studies $[9,40]$ that reported that OA patients who had a better response to glucosamine plus chondroitin sulfate treatment were those with less severe disease but greater cartilage degradation.

HA was found to be more strongly associated with the response to treatment with chondroitin sulfate than CRP. It could be speculated that HA may be more specific than CRP at assessing synovial inflammation. One might argue that the celecoxib treated patients had significantly higher CRP values at baseline and therefore a more severe degree of inflammation, which could have precluded a response to treatment. However, the stratification of patients based on the median value of CRP, which did not differ between treatment subgroups, does not provide support for such a hypothesis. HA has been found to be correlated with symptoms (WOMAC score) and with the degree of radiographic evidence of the disease (Kellgren-Lawrence (KL) grade) [41]. In the present study, patients with lower HA were more responsive to treatment with chondroitin sulfate, which, from the aforementioned findings and those in the review by Singh et al. [41], could mean that patients with less cartilage damage had a better response to this treatment.

The aforementioned data also agree with those from the adipokines, in that patients treated with chondroitin sulfate who had lower baseline levels of adipokines had 
less pronounced cartilage loss and likely less synovial inflammation [42], a finding that is in line with those related to HA. This has potential clinical relevance because leptin and adipsin have been associated not only with synovial inflammation but also with OA progression and cartilage degradation in both knee and hip OA as assessed by qMRI $[24,43,44]$ and with the incidence of total knee replacement [24].

There was also evidence that the level of type II collagen synthesis in OA cartilage, assessed by measuring PIIANP, was clearly associated with response to treatment. The beneficial effect of chondroitin sulfate observed in patients with higher PIIANP values, which is usually indicative of less advanced disease [45], also supports the aforementioned assumption that the response to such treatment may be more pronounced in these patients.

The protective effect of chondroitin sulfate in patients with higher levels of CTX-I, reflecting an increase in the turnover of type I collagen in bone, is interesting and may possibly be related to the positive effect of chondroitin sulfate on bone marrow lesions [8] and on mechanisms related to the remodeling of bone in OA patients $[2,5,46]$.

This study has limitations, the main one being related to the fact that the original study [6] was exploratory and therefore the analysis was performed on the available samples, which were from a limited number of patients. The absence of correction for multiple comparisons is also a study limitation, but should be taken in the context of an exploratory trial. Nevertheless, findings from this study agree with some [8, 47-54], but not all [55-58], previous trials/studies and meta-analyses. Pain could not be considered as a variable, as in the original MOSAIC trial [6] chondroitin sulfate showed a beneficial effect comparable to that of celecoxib. The data on CTX-1 were obtained from serum samples as, unfortunately, urine samples were not available. This could have had an impact on the correlation with other biomarkers. The absence of correlation between CRP and HA was somewhat surprising; the smaller number of samples for CRP could potentially have impacted the results of the analysis. Finally, the use of qMRI to assess the overall cartilage loss over time in knee OA provides a global appreciation of the change in knee cartilage volume in specific anatomical areas and represents a summation of cartilage loss (negative value) and areas of cartilage swelling (positive value), both of which are part of the OA cartilage changes observed during the evolution of knee OA.

\section{Conclusion}

Although this study is exploratory and should be replicated with a larger number of patients to fully confirm the findings, data indicate that higher baseline values of the biomarkers related to collagen metabolism, PIIANP and CTX-1, and those related to cartilage degradation, MMP-1 and MMP-3, and lower baseline values of biomarkers related to inflammation, HA, leptin and adipsin, seem to be related to better response to chondroitin sulfate treatment in patients with knee OA. This is supportive of a potentially better response to treatment with chondroitin sulfate on cartilage volume loss in patients with knee OA with greater levels of cartilage catabolism and/or lower levels of inflammation.

\section{Additional file}

Additional file 1: Table S1. Medial region cartilage volume at baseline according to median value of baseline biomarker levels. Table S2. Medial region cartilage volume at baseline according to median value of baseline biomarker levels per treatment group. (PDF 66 kb)

\section{Abbreviations}

ANCOVA: Analysis of covariance; ATP: According-to-protocol; BMI: Body mass index; CRP: C reactive protein; CTX-1: C-terminal crosslinked telopeptide of type I collagen; ELISA: Enzyme-linked immunosorbent assay; HA: Hyaluronic acid; KL: Kellgren-Lawrence; MDD: Minimum detectable dose; MMP: Matrix metalloproteinase; NSAID: Non-steroidal anti-inflammatory drug; OA: Osteoarthritis; PIIANP: N-terminal propeptide of collagen Ila; qMRI: Quantitative magnetic resonance imaging; SD: Standard deviation; VAS: Visual analog scale; WOMAC: Western Ontario and McMaster Universities Osteoarthritis Index

\section{Acknowledgements}

The authors thank André Pelletier and Josée Thériault for the MRI reading and Virginia Wallis for her assistance with the manuscript preparation.

\section{Funding}

The original trial was funded by Bioibérica SAU, Barcelona, Spain. The sponsor provided all of the study medication free of charge and met the expenses that arose during the course of the study and post hoc analysis. This study was funded by an unrestrictive grant from Bioibérica. The sponsor had no role in the design or conduct of the study, the management, analysis, or interpretation of the data, or the preparation, review, or approval of the manuscript. The sponsor had no access to the data and did not perform any of the study analysis.

\section{Authors' contributions}

JM-P and J-PP designed the study and J-PR participated in the study design. PP was responsible for the collection and integrity of the serum. FM conducted the biomarker experiments and the related data interpretation. FA performed the MRI determination and the related data interpretation. PD performed the statistical analyses and related data interpretation. JM-P, J-PP, J-PR, and PD performed the overall data analysis and interpretation. JM-P and J-PP drafted the manuscript and J-PR assisted with drafting the manuscript. All of the authors read and approved the final manuscript.

\section{Ethics approval and consent to participate}

The original study protocol was approved by a central review board (IRB Institutional Review Board Services, Toronto, ON, Canada) and the institutional review board of the Centre hospitalier de l'Université de Sherbrooke (CHUS), Sherbrooke, QC, Canada. The original trial [6] was conducted in compliance with the ethical principles that have their origin in the Declaration of Helsinki (2000) and are consistent with the "Good Clinical Practice" ICH Tripartite Guideline (January 1997) and the applicable laws and regulations of Canada, whichever afforded the greater protection to the individual. Ethical approval for this post hoc study was obtained with the original study, thus further approval was not required. All patients provided informed consent.

\section{Competing interests}

JM-P and J-PP are consultants for Bioiberica, SAU and shareholders in ArthroLab Inc. J-PR is a consultant for ArthroLab Inc. FA, PP and PD are employees of 
ArthroLab Inc. FM has no conflict of interest. No non-financial conflicts of interest exist for any of the authors.

\section{Publisher's Note}

Springer Nature remains neutral with regard to jurisdictional claims in published maps and institutional affiliations.

\section{Author details}

'Osteoarthritis Research Unit, University of Montreal Hospital Research Centre (CRCHUM), 900 Saint-Denis, Suite R11.412, Montreal, Quebec H2X 0A9, Canada. ${ }^{2}$ Medical Imaging Research \& Development, ArthroLab Inc, Montreal, Quebec, Canada. ${ }^{3}$ ArthroLab Inc, Montreal, Quebec, Canada.

Received: 5 April 2017 Accepted: 27 June 2017

Published online: 20 July 2017

\section{References}

1. Chan PS, Caron JP, Orth MW. Effect of glucosamine and chondroitin sulfate on regulation of gene expression of proteolytic enzymes and their inhibitors in interleukin-1-challenged bovine articular cartilage explants. Am J Vet Res. 2005:66(11):1870-6.

2. Tat SK, Pelletier JP, Vergés J, Montell E, Lajeunesse D, Fahmi H, Lavigne M, Martel-Pelletier J. Chondroitin and glucosamine sulfate in combination decrease the pro-resorptive properties of human osteoarthritis subchondral bone osteoblasts. Arthritis Res Ther. 2007;9:R117.

3. Monfort J, Pelletier J-P, Garcia-Giralt N, Martel-Pelletier J. Biochemical basis of the effect of chondroitin sulfate on osteoarthritis articular tissues. Ann Rheum Dis. 2008;67:735-40.

4. du Souich P, Garcia AG, Verges J, Montell E. Immunomodulatory and antiinflammatory effects of chondroitin sulphate. J Cell Mol Med. 2009;13(8A): 1451-63.

5. Martel-Pelletier J, Kwan Tat S, Pelletier JP. Effects of chondroitin sulfate in the pathophysiology of the osteoarthritic joint: a narrative review. Osteoarthritis Cartilage. 2010;18 Suppl 1:S7-11.

6. Pelletier JP, Raynauld JP, Beaulieu AD, Bessette L, Morin F, de BrumFernandes AJ, Delorme P, Dorais M, Paiement P, Abram F, et al. Chondroitin sulfate efficacy versus celecoxib on knee osteoarthritis structural changes using magnetic resonance imaging: a 2-year multicentre exploratory study. Arthritis Res Ther. 2016;18(1):256.

7. Hochberg MC, Martel-Pelletier J, Monfort J, Moller I, Castillo JR, Arden N, Berenbaum F, Blanco FJ, Conaghan PG, Domenech G, et al. Combined chondroitin sulfate and glucosamine for painful knee osteoarthritis: a multicentre, randomised, double-blind, non-inferiority trial versus celecoxib. Ann Rheum Dis. 2016;75(1):37-44.

8. Wildi LM, Raynauld JP, Martel-Pelletier J, Beaulieu A, Bessette L, Morin F, Abram F, Dorais M, Pelletier JP. Chondroitin sulphate reduces both cartilage volume loss and bone marrow lesions in knee osteoarthritis patients starting as early as 6 months after initiation of therapy: a randomised, double-blind, placebo-controlled pilot study using MRI. Ann Rheum Dis. 2011;70(6):982-9.

9. Martel-Pelletier J, Roubille C, Abram F, Hochberg MC, Dorais M, Delorme P Raynauld JP, Pelletier JP. First-line analysis of the effects of treatment on progression of structural changes in knee osteoarthritis over 24 months: data from the osteoarthritis initiative progression cohort. Ann Rheum Dis. 2015;74(3):547-56.

10. Jordan KM, Arden NK, Doherty M, Bannwarth B, Bijlsma JW, Dieppe P, Gunther K, Hauselmann H, Herrero-Beaumont G, Kaklamanis P, et al. EULAR recommendations 2003: an evidence based approach to the management of knee osteoarthritis: Report of a Task Force of the Standing Committee for International Clinical Studies Including Therapeutic Trials (ESCISIT). Ann Rheum Dis. 2003;62(12):1145-55.

11. Bruyere O, Cooper C, Pelletier JP, Branco J, Luisa Brandi M, Guillemin F, Hochberg MC, Kanis JA, Kvien TK, Martel-Pelletier J, et al. An algorithm recommendation for the management of knee osteoarthritis in Europe and internationally: a report from a task force of the European Society for Clinical and Economic Aspects of Osteoporosis and Osteoarthritis (ESCEO). Semin Arthritis Rheum. 2014;44(3):253-63.

12. Hochberg MC, Altman RD, April KT, Benkhalti M, Guyatt G, McGowan J, Towheed T, Welch V, Wells G, Tugwell P. American College of Rheumatology 2012 recommendations for the use of nonpharmacologic and pharmacologic therapies in osteoarthritis of the hand, hip, and knee. Arthritis Care Res (Hoboken). 2012;64(4):465-74.

13. McAlindon TE, Bannuru RR, Sullivan MC, Arden NK, Berenbaum F, BiermaZeinstra SM, Hawker GA, Henrotin Y, Hunter DJ, Kawaguchi H, et al. OARSI guidelines for the non-surgical management of knee osteoarthritis. Osteoarthritis Cartilage. 2014;22(3):363-88.

14. Lotz M, Martel-Pelletier J, Christiansen C, Brandi ML, Bruyere O, Chapurlat R, Collette J, Cooper C, Giacovelli G, Kanis JA, et al. Value of biomarkers in osteoarthritis: current status and perspectives. Ann Rheum Dis. 2013;72(11):1756-63.

15. Daghestani HN, Kraus VB. Inflammatory biomarkers in osteoarthritis. Osteoarthritis Cartilage. 2015;23(11):1890-6.

16. Hunter DJ, Nevitt M, Losina E, Kraus V. Biomarkers for osteoarthritis: current position and steps towards further validation. Best Pract Res Clin Rheumatol. 2014;28(1):61-71.

17. Kraus VB, Blanco FJ, Englund M, Henrotin Y, Lohmander LS, Losina E, Onnerfjord P, Persiani S. OARSI clinical trials recommendations: soluble biomarker assessments in clinical trials in osteoarthritis. Osteoarthritis Cartilage. 2015;23(5):686-97.

18. Kraus VB, Collins JE, Hargrove D, Losina E, Nevitt M, Katz JN, Wang SX, Sandell LJ, Hoffmann SC, Hunter DJ, et al. Predictive validity of biochemical biomarkers in knee osteoarthritis: data from the FNIH OA Biomarkers Consortium. Ann Rheum Dis. 2016. doi:10.1136/annrheumdis2016-209252.

19. Arden N, Richette P, Cooper C, Bruyere O, Abadie E, Branco J, Brandi ML, Berenbaum F, Clerc C, Dennison E, et al. Can we identify patients with high risk of osteoarthritis progression who will respond to treatment? A focus on biomarkers and frailty. Drugs Aging. 2015;32(7):525-35.

20. Attur M, Krasnokutsky-Samuels S, Samuels J, Abramson SB. Prognostic biomarkers in osteoarthritis. Curr Opin Rheumatol. 2013;25(1):136-44.

21. Kraus VB, Hargrove DE, Hunter DJ, Renner JB, Jordan JM. Establishment of reference intervals for osteoarthritis-related soluble biomarkers: the FNIH/ OARSI OA Biomarkers Consortium. Ann Rheum Dis. 2016. doi:10.1136/ annrheumdis-2016-209253.

22. Pelletier JP, Cooper C, Peterfy C, Reginster JY, Brandi ML, Bruyere O, Chapurlat R, Cicuttini F, Conaghan PG, Doherty $M$, et al. What is the predictive value of MRI for the occurrence of knee replacement surgery in knee osteoarthritis? Ann Rheum Dis. 2013;72(10):1594-604.

23. Pelletier JP, Raynauld JP, Caron J, Mineau F, Abram F, Dorais M, Haraoui B, Choquette D, Martel-Pelletier J. Decrease in serum level of matrix metalloproteinases is predictive of the disease-modifying effect of osteoarthritis drugs assessed by quantitative MRI in patients with knee osteoarthritis. Ann Rheum Dis. 2010;69(12):2095-101.

24. Martel-Pelletier J, Raynauld JP, Dorais M, Abram F, Pelletier JP. The levels of the adipokines adipsin and leptin are associated with knee osteoarthritis progression as assessed by MRI and incidence of total knee replacement in symptomatic osteoarthritis patients: a post hoc analysis. Rheumatology (Oxford). 2016;55(4):680-8.

25. Altman R, Asch E, Bloch D, Bole G, Borenstein D, Brandt K, Christy W, Cooke TD, Greenwald R, Hochberg M, et al. Development of criteria for the classification and reporting of osteoarthritis. Classification of osteoarthritis of the knee. Diagnostic and Therapeutic Criteria Committee of the American Rheumatism Association. Arthritis Rheum. 1986;29(8):1039-49.

26. Raynauld JP, Martel-Pelletier J, Beaulieu A, Bessette L, Morin F, Choquette D, Haraoui B, Abram F, Pelletier JP. An open-label pilot study evaluating by magnetic resonance imaging the potential for a disease-modifying effect of celecoxib compared to a modelized historical control cohort in the treatment of knee osteoarthritis. Semin Arthritis Rheum. 2010;40(3):185-92.

27. Raynauld JP, Martel-Pelletier J, Berthiaume MJ, Labonté F, Beaudoin G, de Guise JA, Bloch DA, Choquette D, Haraoui B, Altman RD, et al. Quantitative magnetic resonance imaging evaluation of knee osteoarthritis progression over two years and correlation with clinical symptoms and radiologic changes. Arthritis Rheum. 2004;50:476-87.

28. Kauffmann C, Gravel P, Godbout B, Gravel A, Beaudoin G, Raynauld JP, MartelPelletier J, Pelletier JP, de Guise JA. Computer-aided method for quantification of cartilage thickness and volume changes using MRl: validation study using a synthetic model. IEEE Trans Biomed Eng. 2003;50(8):978-88.

29. Pelletier JP, Raynauld JP, Abram F, Haraoui B, Choquette D, Martel-Pelletier J. A new non-invasive method to assess synovitis severity in relation to symptoms and cartilage volume loss in knee osteoarthritis patients using MRI. Osteoarthritis Cartilage. 2008;16 Suppl 3:S8-13. 
30. Li W, Abram F, Pelletier JP, Raynauld JP, Dorais M, d'Anjou MA, MartelPelletier J. Fully automated system for the quantification of human osteoarthritic knee joint effusion volume using magnetic resonance imaging. Arthritis Res Ther. 2010;12(5):R173.

31. Raynauld JP, Martel-Pelletier J, Berthiaume MJ, Beaudoin G, Choquette D, Haraoui B, Tannenbaum H, Meyer JM, Beary JF, Cline GA, et al. Long term evaluation of disease progression through the quantitative magnetic resonance imaging of symptomatic knee osteoarthritis patients: correlation with clinical symptoms and radiographic changes. Arthritis Res Ther. 2006;8(1):R21.

32. Wildi LM, Martel-Pelletier J, Abram F, Moser T, Raynauld JP, Pelletier JP. Assessment of cartilage changes over time in knee osteoarthritis diseasemodifying osteoarthritis drug trials using semiquantitative and quantitative methods: pros and cons. Arthritis Care Res (Hoboken). 2013;65(5):686-94.

33. Koskinen A, Vuolteenaho K, Nieminen R, Moilanen T, Moilanen E. Leptin enhances MMP-1, MMP-3 and MMP-13 production in human osteoarthritic cartilage and correlates with MMP-1 and MMP-3 in synovial fluid from OA patients. Clin Exp Rheumatol. 2011;29(1):57-64.

34. Bay-Jensen AC, Reker D, Kjelgaard-Petersen CF, Mobasheri A, Karsdal MA, Ladel C, Henrotin Y, Thudium CS. Osteoarthritis year in review 2015: soluble biomarkers and the BIPED criteria. Osteoarthritis Cartilage. 2016;24(1):9-20.

35. Smith JW, Martins TB, Gopez E, Johnson T, Hill HR, Rosenberg TD. Significance of C-reactive protein in osteoarthritis and total knee arthroplasty outcomes. Ther Adv Musculoskelet Dis. 2012;4(5):315-25.

36. Sharif M, Shepstone L, Elson CJ, Dieppe PA, Kirwan JR. Increased serum C reactive protein may reflect events that precede radiographic progression in osteoarthritis of the knee. Ann Rheum Dis. 2000;59:71-4.

37. Wang $X$, Hunter $D, X u$ J, Ding C. Metabolic triggered inflammation in osteoarthritis. Osteoarthritis Cartilage. 2015;23(1):22-30.

38. Saberi Hosnijeh F, Siebuhr AS, Uitterlinden AG, Oei EH, Hofman A, Karsdal MA, Bierma-Zeinstra SM, Bay-Jensen AC, van Meurs JB. Association between biomarkers of tissue inflammation and progression of osteoarthritis: evidence from the Rotterdam study cohort. Arthritis Res Ther. 2015;18:81.

39. Jin X, Beguerie JR, Zhang W, Blizzard L, Otahal P, Jones G, Ding C. Circulating $C$ reactive protein in osteoarthritis: a systematic review and meta-analysis. Ann Rheum Dis. 2015;74(4):703-10.

40. Roubille C, Martel-Pelletier J, Abram F, Dorais M, Delorme P, Raynauld JP, Pelletier JP. Impact of disease treatments on the progression of knee osteoarthritis structural changes related to meniscal extrusion: Data from the OAl progression cohort. Semin Arthritis Rheum. 2015;45(3):257-67.

41. Singh S, Kumar D, Sharma NR. Role of hyaluronic acid in early diagnosis of knee osteoarthritis. J Clin Diagn Res. 2014;8(12):LC04-07.

42. de Boer TN, van Spil WE, Huisman AM, Polak AA, Bijlsma JW, Lafeber FP, Mastbergen SC. Serum adipokines in osteoarthritis; comparison with controls and relationship with local parameters of synovial inflammation and cartilage damage. Osteoarthritis Cartilage. 2012;20(8):846-53.

43. Stannus OP, Jones G, Quinn SJ, Cicuttini FM, Dore D, Ding C. The association between leptin, interleukin-6, and hip radiographic osteoarthritis in older people: a cross-sectional study. Arthritis Res Ther. 2010;12(3):R95

44. Stannus OP, Cao Y, Antony B, Blizzard L, Cicuttini F, Jones G, Ding C. Crosssectional and longitudinal associations between circulating leptin and knee cartilage thickness in older adults. Ann Rheum Dis. 2015:74(1):82-8.

45. Berry PA, Maciewicz RA, Wluka AE, Downey-Jones MD, Forbes A, Hellawell CJ, Cicuttini FM. Relationship of serum markers of cartilage metabolism to imaging and clinical outcome measures of knee joint structure. Ann Rheum Dis. 2010;69(10):1816-22.

46. Raynauld JP, Pelletier JP, Delorme P, Dodin P, Abram F, Martel-Pelletier J. Bone curvature changes can predict in knee osteoarthritis the impact of treatment on cartilage volume loss: data from a two-year clinical trial. Rheumatology (Oxford). 2017;56(6):989-98.

47. Uebelhart D, Malaise M, Marcolongo R, DeVathaire F, Piperno M, Mailleux E, Fioravanti A, Matoso L, Vignon E. Intermittent treatment of knee osteoarthritis with oral chondroitin sulfate: a one-year, randomized, double-blind, multicenter study versus placebo. Osteoarthritis Cartilage. 2004;12(4):269-76.

48. Michel BA, Stucki G, Frey D, De Vathaire F, Vignon E, Bruehlmann P, Uebelhart D. Chondroitins 4 and 6 sulfate in osteoarthritis of the knee: a randomized, controlled trial. Arthritis Rheum. 2005;52(3):779-86.

49. Kahan A, Uebelhart D, De Vathaire F, Delmas PD, Reginster JY. Long-term effects of chondroitins 4 and 6 sulfate on knee osteoarthritis: the study on osteoarthritis progression prevention, a two-year, randomized, double-blind, placebo-controlled trial. Arthritis Rheum. 2009:60(2):524-33.
50. Hochberg MC. Structure-modifying effects of chondroitin sulfate in knee osteoarthritis: an updated meta-analysis of randomized placebo-controlled trials of 2-year duration. Osteoarthritis Cartilage. 2010;18 Suppl 1:S28-31.

51. Lee $\mathrm{YH}$, Woo JH, Choi SJ, Ji JD, Song GG. Effect of glucosamine or chondroitin sulfate on the osteoarthritis progression: a meta-analysis. Rheumatol Int. 2010;30(3):357-63.

52. Hochberg M, Chevalier X, Henrotin Y, Hunter DJ, Uebelhart D. Symptom and structure modification in osteoarthritis with pharmaceutical-grade chondroitin sulfate: what's the evidence? Curr Med Res Opin. 2013;29(3):259-67.

53. Gallagher B, Tjoumakaris FP, Harwood MI, Good RP, Ciccotti MG, Freedman $\mathrm{KB}$. Chondroprotection and the prevention of osteoarthritis progression of the knee: a systematic review of treatment agents. Am J Sports Med. 2015; 43(3):734-44.

54. Singh JA, Noorbaloochi S, MacDonald R, Maxwell LJ. Chondroitin for osteoarthritis. Cochrane Database Syst Rev. 2015;1:CD005614.

55. Richy F, Bruyere O, Ethgen O, Cucherat M, Henrotin Y, Reginster JY. Structural and symptomatic efficacy of glucosamine and chondroitin in knee osteoarthritis: a comprehensive meta-analysis. Arch Intern Med. 2003;163(13):1514-22.

56. Clegg DO, Reda DJ, Harris CL, Klein MA, O'Dell JR, Hooper MM, Bradley JD, Bingham 3rd CO, Weisman MH, Jackson CG, et al. Glucosamine, chondroitin sulfate, and the two in combination for painful knee osteoarthritis. N Engl J Med. 2006:354(8):795-808.

57. Reichenbach S, Sterchi R, Scherer M, Trelle S, Burgi E, Burgi U, Dieppe PA, Juni P. Meta-analysis: chondroitin for osteoarthritis of the knee or hip. Ann Intern Med. 2007;146(8):580-90.

58. Wandel S, Juni P, Tendal B, Nuesch E, Villiger PM, Welton NJ, Reichenbach S, Trelle S. Effects of glucosamine, chondroitin, or placebo in patients with osteoarthritis of hip or knee: network meta-analysis. BMJ. 2010;341:c4675.

\section{Submit your next manuscript to BioMed Central and we will help you at every step:}

- We accept pre-submission inquiries

- Our selector tool helps you to find the most relevant journal

- We provide round the clock customer support

- Convenient online submission

- Thorough peer review

- Inclusion in PubMed and all major indexing services

- Maximum visibility for your research

Submit your manuscript at www.biomedcentral.com/submit
Ciomed Central 